\title{
BLAUES LICHT UND SEINE WIRKUNG - NEUESTE FORSCHUNGSERGEBNISSE
}

\section{Liebe Kolleginnen und Kollegen,}

„19-4052 Classic Blue“ nennt sich jener Farbton, den das Farbunternehmen Pantone aus den USA zur Farbe des Jahres 2020 kürte. Der Farbton steht für Ruhe, Zuverlässigkeit und Stabilität. Nun belegt eine interessante wissenschaftliche Studie, welche in der renommierten Zeitschrift Neurobiology of Disease (IF 5,227) im Jahr 2020 (Killgore WDS et al.) publiziert wurde, erneut die Wirkung von blauem Licht. Ein Forscherteam der Universität von Arizona (Tucson) konnte zeigen, dass eine Blaulicht-Expositionstherapie am frühen Morgen den Heilungsprozess von Menschen, die von leichten traumatischen Hirnverletzungen betroffen sind, unterstützen kann. Blaues Licht unterdrückt die Produktion von Melatonin. Wenn man morgens blauem Licht ausgesetzt ist, verschiebt sich die biologische Uhr, sodass am Abend die Produktion früher einsetzt und dies führt zu einer besseren Schlafqualität. Auch unser Forschungsteam an der Medizinischen Universität in Graz konnte bereits 2013 mit experimentellen Untersuchungen zur Thematik beitragen. D. Litscher u. Mitarb. konnten nachweisen (Evid Based Complement Alternat Med; IF 2,175), dass es sehr wohl unterschiedliche Effekte auf verschiedene Farbstimulationen (z.B. blau und rot) gibt.

Alle Autoren sind sich einig: Die meisten Menschen, ob verletzt oder gesund, können von einer zeitlich korrekt

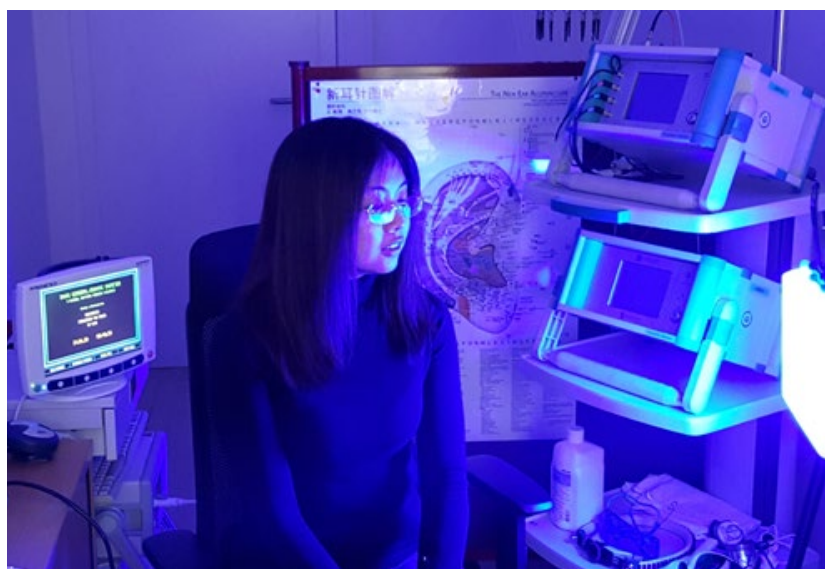

Abb. 1: Gastwissenschaftlerin Yan Yang testet im Jänner 2020 ein blaues Lichtstimulationsgerät am TCM-Zentrum (Chairman: Gerhard Litscher) der Medizinischen Universität Graz

durchgeführten morgendlichen Belichtung mit blauem Licht profitieren, eine Theorie, die auch mit weiteren zukünftigen Studien belegt werden soll. Mehr dazu auf dem nächsten, dem bereits 20. ISLA-Kongress in Beverungen, Deutschland (14.-16. Mai 2020).

Wir wünschen Ihnen allen ein weiterhin erfolgreiches Jahr 2020.

Gerhard Litscher

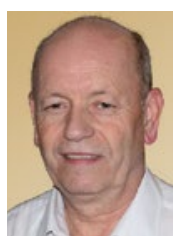

Dr. med. Dipl. Chem. Michael Weber

President for Medical and Clinical Applications der International Society for Medical Laser Applications (ISLAtranscontinental)

Sohnreystrasse 4

37697 Lauenförde

Internet: www.isla-laser.org

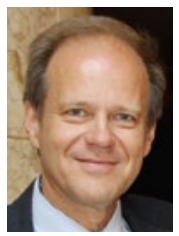

Univ.-Prof. Prof. h.c. DI Dr. techn.

Dr. scient. med. Gerhard Litscher

President for Science and Research der International Society for Medical Laser Applications (ISLAtranscontinental)

Medizinische Universität Graz, Auenbruggerplatz 39, EG19, A-8036 Graz, Tel: +43 316/385-83907

E-Mail:gerhard.litscher@medunigraz.at,Internet:http://litscher.info 